\title{
Le département de l'Eure sous la République directoriale
}

André Goudeau

\section{(2) OpenEdition \\ 12 Journals}

Édition électronique

URL : https://journals.openedition.org/ahrf/11881

DOI : 10.4000/ahrf.11881

ISSN : 1952-403X

\section{Éditeur :}

Armand Colin, Société des études robespierristes

\section{Édition imprimée}

Date de publication : 1 décembre 2010

Pagination : 111-120

ISBN : 978-2-200-92634-2

ISSN : 0003-4436

\section{Référence électronique}

André Goudeau, «Le département de l'Eure sous la République directoriale », Annales historiques de la Révolution française [En ligne], 362 | octobre-décembre 2010, mis en ligne le 01 décembre 2013, consulté le 23 avril 2022. URL : http://journals.openedition.org/ahrf/11881 ; DOI : https://doi.org/ 10.4000/ahrf.11881 


\section{LE DÉPARTEMENT DE L'EURE SOUS LA RÉPUBLIQUE DIRECTORIALE}

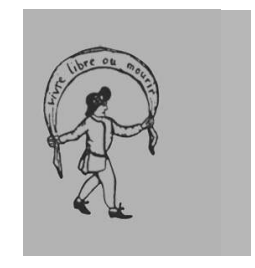

André GOUDEAU

Étudier la période directoriale dans le cadre d'un département, tel est le propos de cette thèse ${ }^{1}$. Le territoire sur lequel a porté la recherche peut sembler restreint mais le cadre départemental apparaît néanmoins comme pertinent et s'impose au chercheur qui souhaite travailler sur la Révolution française. En effet, à partir de 1790, cette nouvelle circonscription administrative a constitué un espace cohérent où se déroulait une vie politique locale ayant son rythme propre, souvent différent de celui des événements parisiens.

Cette monographie départementale vient donc s'ajouter à d'autres traitant du Directoire, celles-ci n'étant d'ailleurs pas si nombreuses. Après l'ouvrage pionnier de Marcel Reinhard consacré à la Sarthe et l'étude de Jean-René Suratteau sur le Mont-Terrible, d'autres travaux ont porté sur des départements, en particulier trois études analysant le régime directorial dans la Meurthe, le Puy-de-Dôme et l'Orne dont les auteurs sont respectivement Pierre Clémendot, Philippe Bourdin et Sylvie DenysBlondeau. Toutes ces monographies ont permis de mettre en avant tel ou tel caractère particulier, venant ainsi compléter, corroborer ou infirmer les recherches menées au niveau national.

Notre thèse s'efforce donc de voir comment l'Eure a vécu les diverses mutations conduites sous le Directoire, en tenant compte des

(1) Thèse de doctorat soutenue devant l'Université de Rouen, le 28 septembre 2009. Le jury était composé de : M. Michel Biard, Directeur de recherche, Professeur à l'Université de Rouen, M. Bernard Bodinier, Professeur émérite à l'IUFM de Rouen, M. Philippe Bourdin, Professeur à l'Université Blaise-Pascal, Clermont-Ferrand, M. Bernard Gainot, Maître de conférences HDR à l'Université de Paris I Panthéon-Sorbonne et M. Éric Wauters, Professeur à l'Université du Havre. 
spécificités de ce département. Proche de Paris, il n'a certes pas joué un rôle de tout premier plan durant les premières années de la Révolution, mais il était forcément sensible au déroulement du processus révolutionnaire dans la capitale. Les communications relativement faciles par la vallée de la Seine favorisaient une circulation rapide de l'information, les nouvelles de Paris étant connues dès le lendemain à Évreux. Nous nous sommes donc demandé si ce voisinage avait eu une influence sur la vie politique du département durant la période directoriale et si les décisions du pouvoir central y furent mises en œuvre rapidement par le nouveau commissaire central, institué par la Constitution de l'an III et nommé par le Directoire exécutif.

Autre conséquence de cette proximité avec la capitale, l'Eure contribuait à l'approvisionnement de Paris en céréales, parfois au détriment de la population locale. La question des « subsistances » fut d'ailleurs une préoccupation constante des autorités révolutionnaires qui ne purent empêcher de fréquentes émeutes. Durant l'hiver de l'an III, suite à une mauvaise récolte et à l'abandon du Maximum, ce mécontentement se manifesta à nouveau à l'encontre du représentant Bernier, envoyé en mission dans l'Eure. Comment l'administration centrale allait-elle affronter ce problème durant la période directoriale, d'autant que, l'autorité des districts ayant disparu, les nouvelles municipalités de canton risquaient de se trouver désarmées devant le refus de vente des cultivateurs?

Inquiet de la pression exercée par les sans-culottes parisiens sur le processus révolutionnaire, l'Eure avait rejoint le fédéralisme normand, sous l'influence de Buzot, en juin 1793. Cette dissidence, si elle ne concernait guère la population, avait été déclenchée par des élus au conseil général du département et aux districts. Bien que s'étant rétractés, nombre de ces administrateurs, emprisonnés à la demande des comités de surveillance, furent libérés après le 9 Thermidor avant de devenir par la suite les cadres du régime directorial. Marqué par l'épisode fédéraliste, ce personnel politique départemental «thermidorien», certes républicain, resta ensuite foncièrement hostile aux « anarchistes ». Le clivage hérité du fédéralisme allait-il rendre impossible l'union des républicains sous le Directoire?

L'Eure avait disposé sous le Gouvernement révolutionnaire d'un réseau dense de sociétés jacobines. Rongées par les divisions et l'absentéisme, ces sociétés populaires, soumises aux pressions des éléments antiterroristes, virent leurs effectifs diminuer après le 9 Thermidor et la plupart d'entre elles disparurent au cours du premier semestre 1795. Nous nous sommes demandés si l'on allait assister au renouveau de cette 
politisation sous le Directoire, le jacobinisme de l'an II renaissant sous la forme d'un « néo-jacobinisme », défenseur intransigeant du « républicanisme ». Les deux frères Lindet, députés montagnards originaires de Bernay, qui se situaient dans cette mouvance et disposaient de réseaux dans le département, allaient-ils poursuivre leur action sous le Directoire et sous quelle forme?

Proche de la capitale et donc soumise à l'influence parisienne, l'Eure se trouvait aussi, par les cantons bocagers du Pays d'Ouche, en limite de l'Ouest rebelle et chouan de la Normandie orientale. Le département dont les massifs forestiers constituaient autant de refuges, fut donc affecté par la chouannerie normande, relayée par un brigandage endémique qui tentait de se donner des couleurs politiques. Les autorités du département durent réagir face à cette " malveillance » et le maintien de l'ordre constitua pour elles une préoccupation constante.

Pour présenter l'Eure sous la République directoriale, nous avons pu faire appel à des sources relativement riches, tant au niveau local qu'à l'échelon national. Aux archives départementales, sont en effet conservées les délibérations de l'administration centrale ainsi que la correspondance du commissaire central avec ses collègues des cantons ainsi qu'avec les ministres. Les procès-verbaux des assemblées électorales tenues au chef-lieu y sont disponibles. Si les listes d'électeurs de l'an IV, de l'an VI et de l'an VII sont conservées, en revanche, celle des électeurs de l'an $\mathrm{V}$ manque et il a donc fallu la reconstituer partiellement à partir de divers documents. Incomplets ou même pratiquement inexistants pour vingt-quatre cantons, les papiers des administrations municipales peuvent être complétés par leurs registres de délibérations, rédigés avec plus ou moins de soin, mais restant très utiles.

La confrontation des archives des administrations locales et de celles du pouvoir central s'est avérée nécessaire. Les dossiers conservés aux Archives nationales nous ont permis de compléter utilement la documentation trouvée à Évreux, d'apprécier comment le Directoire exécutif ou les ministres arbitraient certains conflits politiques locaux et de voir quel regard portait le pouvoir central sur ce département. On y a aussi trouvé d'utiles informations sur la chouannerie, le brigandage et les moyens mis en œuvre par les autorités locales afin de les combattre. Les deux comptes rendus de gestion imprimés, publiés par l'administration centrale en l'an V et l'an VI ont constitué également des documents précieux puisque les administrateurs s'efforçaient d'y exposer et d'y justifier leur politique. Malheureusement, les comptes rendus décadaires ou mensuels auxquels étaient tenus les commissaires centraux manquent pour la 
partie la plus importante de la période. On n'en trouve qu'à la fin du Directoire, Thomas Lindet s'astreignant à cet exercice lorsqu'il eut la charge du commissariat.

Deux sources imprimées nous ont été d'une grande utilité : le journal tenu à Évreux par Nicolas Rogue dans lequel il notait scrupuleusement ce qui se passait sous ses yeux et le Bulletin de l'Eure du journaliste néo-jacobin Touquet qui parut à Évreux pendant dix-sept mois environ, du 15 frimaire an V (5 décembre 1796) au 21 germinal an VI (10 avril 1798).

Pour tenter de saisir l'originalité de la période directoriale dans l'Eure, la thèse suit un plan à la fois chronologique et thématique, s'efforçant de traiter d'une " histoire totale », présentant non seulement la vie politique et administrative du département mais décrivant également les évolutions intervenues dans les domaines culturel, religieux et économique entre 1795 et 1799 ; elle comporte cinq parties.

Est tout d'abord dressé un état des lieux, présentant le département au point de vue physique, humain et historique en 1795 puis décrivant la mise en place des nouvelles institutions, après l'adoption de la Constitution de l'an III et les élections de l'an IV. La seconde partie s'efforce de montrer comment le commissaire Crochon, l'administration centrale et les nouvelles municipalités cantonales ont fait face à leurs tâches administratives, affrontant la crise de subsistances de l'an IV, réprimant la chouannerie et le brigandage tout en étant confrontés à la renaissance du jacobinisme. La thèse analyse ensuite la période s'ouvrant avec les élections de l'an $\mathrm{V}$ marquées par un succès royaliste et se terminant par celles de germinal VI, gagnées par les républicains, après de nombreuses scissions au sein des assemblées cantonales. Cette séquence particulièrement tourmentée, fut marquée, entre les deux scrutins, par l'assassinat de l'ancien président du département, les destitutions faisant suite à Fructidor ainsi que par une contestation de plus en plus vive de l'administration centrale et du commissaire Crochon par le journaliste Touquet. Le scrutin de 1798 vit l'élection de Crochon aux Cinq-Cents et la nomination de Savary comme commissaire.

La quatrième partie, intitulée "Vers une nouvelle citoyenneté » nous a permis de présenter l'œuvre des autorités départementales dans le domaine de l'éducation (avec en particulier la mise en place de l'école centrale), dans celui des fêtes, des religions républicaines ainsi qu'en faveur de la modernisation de l'économie. Parallèlement, nous avons examiné la réception de ce message civique dans la population allant de pair avec la lutte contre le « fanatisme », notamment après Fructidor, sans négliger les 
efforts du clergé constitutionnel pour essayer de reconstruire le diocèse. Enfin, la dernière partie traite de la fin du Directoire. Après les élections de l'an VII et l'éphémère mandat de François Rever au commissariat central, Thomas Lindet s'efforça de relancer la lutte contre la chouannerie, cherchant à s'appuyer sur les néo-jacobins, mais ses efforts furent interrompus par Brumaire.

Quels enseignements tirer de cette recherche? Entre l'an IV et l'an VIII, l'Eure, département modéré, suivit globalement l'évolution du régime sans faire preuve d'une très grande originalité. Toutefois, on peut malgré tout souligner certains traits particuliers de ce département durant l'épisode directorial.

Tout d'abord, l'Eure fut constamment en proie à des désordres durant la période. En effet, il a ressenti les contrecoups des deux guerres chouannes se déroulant dans les départements de Normandie orientale. Cette agitation, souvent menée par des déserteurs et soutenue par des brigands cherchant à donner une coloration politique à leurs méfaits, se développa surtout dans le sud-ouest du département. Pour faire face à ces troubles politiques complétés par un brigandage endémique, notamment celui des « chauffeurs » de la bande de Robillard, les autorités disposaient de moyens militaires insuffisants qu'il s'agisse de la gendarmerie, trop peu nombreuse, de la garde nationale, mal organisée dans les cantons, ou de forces militaires aux effectifs insuffisants. Le recours aux espions, entrepris par les commissaires Rever et Lindet à la fin de la période, ne permit pas d'obtenir de réels succès.

À l'autre extrémité de l'échiquier politique, un courant néo-jacobin se manifesta dans le département sous le Directoire et prit son autonomie après Fructidor, disposant de plusieurs atouts. Tout d'abord un journal, le Bulletin de l'Eure rédigé par Touquet, imprimeur et journaliste de talent, qui, après avoir prôné l'union des républicains autour de la Constitution de l'an III, se lança dans une sévère dénonciation de l'administration centrale et du commissaire Crochon, jugeant leur « républicanisme » douteux. Ce périodique était lu dans la quinzaine de cercles constitutionnels créés dans le département, héritiers de la pratique jacobine des nombreuses sociétés populaires qui s'étaient constituées en l'an II. Ces cercles fonctionnèrent surtout dans les chefs-lieux de cantons ruraux, les militants néo-jacobins de ces bourgs patriotes retrouvant, après Fructidor, le chemin de la défense républicaine, multipliant les adresses au Directoire et au Corps législatif et s'en prenant aux administrateurs départementaux trop enclins aux compromis avec les adversaires de la République. Le Bulletin de l'Eure ayant disparu en floréal an VI, ce courant manqua alors 
de moyens d'expression avant de se reconnaître dans l'action du commissaire Thomas Lindet à la veille de Brumaire.

Figures du néo-jacobinisme, les frères Lindet, auréolés par leur action passée, ne furent pourtant pas les meneurs du mouvement. Robert qui, d'emblée, refusa le poste de commissaire central, fut accusé d'avoir participé à la conspiration des Égaux mais n'eut aucune action politique réelle dans le département. Une fois blanchi, les électeurs le choisirent comme député en l'an VI mais il fut presque aussitôt « floréalisé », ne revenant sur le devant de la scène qu'au cours de l'été 1799, lorsqu'il accepta le poste de ministre des Finances. Quant à Thomas, élu aux Anciens en l'an IV, il se consacra d'abord à la défense de son frère jugé à Vendôme puis intrigua auprès des ministres pour obtenir des destitutions et pour tenter de faire nommer des « républicains avancés » dans les municipalités cantonales. Comme Robert, il revint aux affaires durant l'été 1799, acceptant alors le poste de commissaire central de l'Eure dans une situation quasi désespérée, marquée par le renouveau des violences perpétrées par les brigands et les chouans.

Thomas Lindet avait succédé à Rever, qui fut en floréal an VII, un éphémère commissaire central après avoir été, en tant que membre du jury d'instruction, l'inlassable animateur de l'école centrale, intervenant auprès des administrateurs pour que celle-ci dispose de locaux corrects, assurant la fonction de bibliothécaire et de professeur d'arts mécaniques. Cet établissement scolaire installé à Évreux fut particulièrement novateur, comme le remarqua d'ailleurs Ferdinand Buisson. On y pratiquait une pédagogie expérimentale dans le cabinet de physique, on y observait les plantes dans le jardin botanique mais, surtout, on y organisait des sorties pédagogiques tout à fait originales. Rever réussit à doter l'école centrale d'un pensionnat, il organisa l'établissement en fonction d'un règlement hérité des Lumières et, dans ses écrits, il souhaitait la mise en place d'une « instruction publique [...] véritablement gratuite ». Malheureusement, l'école centrale ne scolarisa qu'une frange très réduite de la jeunesse, ne formant qu'une modeste élite républicaine.

Comme l'enseignement, le théâtre avait pour fonction d'éduquer le citoyen et de développer son civisme. Le département de l'Eure, proche de la capitale, reçut de nombreuses troupes de comédiens et connut de l'an IV à l'an VIII, une activité théâtrale fort riche, surtout à Évreux. Les comédies légères $\mathrm{du}$ XVIII ${ }^{\mathrm{e}}$ siècle retrouvaient les faveurs du public mais les pièces à caractère politique n'avaient pas disparu. Des œuvres de circonstance se déchaînant contre les Jacobins étaient programmées, certaines répliques provoquant dans le public des réactions contradictoires. 
Ces spectacles, inspirés par l'actualité, étaient autorisés, ce qui provoquait la colère des néo-jacobins et tout particulièrement de leur porteparole, le journaliste Touquet, qui réclamait la mise à l'affiche de pièces qui « ravivent l'esprit public ». Ici comme ailleurs, le théâtre fut source de polémiques sans que, pour autant, ces conflits ne dégénèrent en affrontements violents. Une œuvre, La jeunesse du duc de Richelieu de PineauxDuval et de Montvel, fut bien interdite, mais parce que les administrateurs la jugeaient « immorale».

Outre l'enseignement et le théâtre, les fêtes et les religions républicaines devaient alimenter et renforcer le civisme de la population. Le cycle des fêtes citoyennes était mis en place pour rythmer la vie de la population. Dans l'Eure, le manque d'argent, de motivation politique, l'indifférence grandissante de nombre de citoyens conduisirent bien des municipalités à ne choisir que quelques dates seulement, voire à produire de faux procèsverbaux. Comme partout, les autorités eurent beaucoup de mal à imposer le calendrier républicain et, dans l'Eure, surtout en milieu rural, les habitants continuaient à respecter le dimanche et les jours de fêtes consacrés aux saints patrons. Les cultes civiques eurent-ils plus de succès? La Théophilanthropie recruta des adeptes à Bernay et à Verneuil et semble y avoir connu un certain succès avant d'entrer en concurrence avec le culte décadaire institué en l'an VI, comme le note Albert Mathiez. La cohabitation des cultes décadaire et catholique dans un même « temple »-l'église paroissiale - provoqua souvent des incidents.

Ces cultes républicains entraient en conflit avec la religion catholique dont les prêtres étaient divisés entre « romains » et " gallicans ». Dans l'Eure, le clergé, en majorité jureur, avait plutôt accueilli favorablement les réformes de l'Église dans les premières années de la Révolution. La tentative de politique anticatholique menée par l'administration centrale après Fructidor échoua car elle resta globalement impopulaire dans un département qui n'était pas particulièrement «fanatique ». Cette politique fut appuyée par d'anciens prêtres devenus très hostiles au catholicisme, qu'il s'agisse de Lecerf ou de Rioust. Le clergé constitutionnel - ou ce qu'il en restait - était prêt à fait preuve de civisme et de soumission à l'autorité politique, ce qui aurait dû permettre une «coexistence pacifique » dans le cadre de cette première séparation de l'Église et de l'État. Les efforts des gallicans pour reconstruire le diocèse ressemblèrent à ceux rencontrés dans d'autres diocèses normands: désignation du conseil presbytéral, réunion d'un synode, préparation du concile. Notons toutefois l'originalité que constitue le Journal du Bon sens publié par l'abbé Moulis, délégué au concile national, qui aurait bien voulu devenir 
évêque mais qui ne fut pas désigné. Le Bulletin de l'Eure polémiquait fréquemment avec le périodique rédigé par Moulis qui, malgré une faible diffusion, eut certainement une réelle influence dans le diocèse. Bien que souvent trop théorique, il contribua à la diffusion des idées et des thèmes de réflexion des "réunis » et surtout, il permit d'établir des liens entre des ecclésiastiques souvent isolés. D'ailleurs, en fructidor an $\mathrm{V}$, faisant le bilan de l'action des "évêques réunis » lors du concile national, l'abbé Grégoire mentionna le rôle du Journal du bons sens à Évreux.

Pour faire fonctionner les institutions mises en place au niveau local et départemental, le régime directorial devait disposer d'un personnel politique nombreux, formant une sorte de « classe politique [départementale] en formation » pour reprendre l'expression de Sylvie Denis-Blondeau, " classe politique » composée des électeurs choisis dans les assemblées primaires, des commissaires cantonaux et membres de municipalités de canton, intéressés à des degrés divers par les affaires publiques.

Ce groupe social constituait un vivier dans lequel étaient choisis les administrateurs du département et les députés. Il existait en effet une sorte de cursus politico-administratif permettant de s'élever dans la hiérarchie des fonctions. Pour devenir député, il était bon d'avoir déjà siégé dans une assemblée révolutionnaire, en l'occurrence à la Convention nationale comme Savary, Thomas Lindet ou Topsent. L'accession au commissariat central ou à l'administration départementale constituait une première étape, prédisposant souvent à la députation. L'itinéraire de Crochon résume d'ailleurs bien cette promotion. Procureur syndic du district de Pont-Audemer, il devint commissaire central avant d'être élu député aux Cinq-Cents en 1798. Il siégera ensuite au Corps législatif de 1799 à 1802 et deviendra maire de Pont-Audemer de 1830 à 1842. Le parcours personnel de Crochon illustre bien la naissance de cette « classe politique » sous le Directoire, préfigurant les notables qui formeront ensuite l'assise du régime napoléonien puis de la monarchie constitutionnelle.

À travers l'étude du département de l'Eure, cette thèse entend se situer dans le cadre du renouvellement historiographique intervenu récemment au sujet de la République directoriale. Ces quatre années, de 1795 à 1799 - soit près de la moitié de la décennie révolutionnaire -, au cours desquelles la France s'est efforcée de « terminer la Révolution », expérimentant un régime républicain constitutionnel, ont longtemps été considérées par les historiens comme une époque terne, période de médiocrité, " interrègne sans éclat », auquel étaient associés des images négatives. Pourtant, durant le Directoire, derrière le désordre apparent, les coups d'État, l'enrichissement et la frivolité de la bourgeoisie, s'opère une 
expérimentation politique intéressante avec des élections annuelles, une première tentative de laïcisation de l'espace public et des innovations institutionnelles au niveau local, comme les municipalités de canton ou la mise en place du commissaire central. En ce sens, Le Directoire constitue un jalon, contribuant à préparer les futures expériences républicaines dans la France du XIX ${ }^{\mathrm{e}}$ siècle.

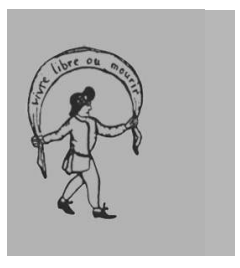

André Goudeau amt.goudeau@wanadoo.fr 